\title{
Characterization of the HCl-HBr-HI gas absorption cell for GIANO- TNG
}

\author{
Francesco D'Amato ${ }^{\mathrm{a}}$, Silvia Viciani ${ }^{\mathrm{a}}$, Ernesto Oliva ${ }^{* \mathrm{~b}}$, Livia Origlia ${ }^{\mathrm{c}}$, Iacopo Mochi ${ }^{\mathrm{b}}$ \\ ${ }^{\mathrm{a}} \mathrm{CNR}$ - Istituto Nazionale di Ottica Applicata, L.go E. Fermi 6, 50125 Firenze, Italy; ${ }^{\mathrm{b}}$ Osservatorio \\ Astrofisico di Arcetri, L.go E. Fermi 5, 50125 Firenze, Italy; ${ }^{\circ}$ Osservatorio Astronomico di Bologna, \\ Via Ranzani 1, 40127 Bologna, Italy
}

\begin{abstract}
GIANO is an high resolution cross-dispersed spectrometer operating at near IR wavelengths (0.9-2.5 microns) which will be soon commissioned at the 3.6m TNG Italian telescope in La Palma. One of its most ambitious aims is searching for earth-like planets with habitable conditions around very cool main sequence stars. This requires measurements of radial velocities with accuracies of a few $\mathrm{m} / \mathrm{s}$ which can be achieved by means of a gas absorption cell containing a mixture of the halogen-hydrates $\mathrm{HCl}, \mathrm{HBr}$ and $\mathrm{HI}$. We present here the results of the laboratory work for the construction and characterization of such cell.
\end{abstract}

Keywords: Ground based infrared instrumentation, infrared spectrometers, Doppler measurements

\section{INTRODUCTION}

GIANO is an IR cross-dispersed spectrometer designed to achieve high resolution (with a resolving power up to $\mathrm{R}=$ $\Delta \lambda / \lambda=50000$ ) and wide band coverage (a quasi complete $0.9-2.5 \mu \mathrm{m}$ range in a single shot). It will be permanently mounted and available at one of the Nasmyth foci of the Telescopio Nazionale Galileo (TNG) located at Roque de Los Muchachos Observatory (ORM), in La Palma (Spain).

One of the primary scientific targets of GIANO is the search of earth-like planets with habitable conditions around very cool main sequence stars, by means of radial velocity measurements with very high accuracy, comparable to those presently achieved at optical wavelengths ${ }^{1}$.

In order to obtain accuracy of few $\mathrm{m} / \mathrm{s}$, a known absorption spectrum must be superimposed to the absorptions due to interstellar matter. This spectrum should contain several ( 200), resolvable, homogeneously distributed lines. The solution is to insert along the optical line a gas absorbtion cell, which must fulfill several costraints. All these costraints have been analyzed and discussed in a previous paper ${ }^{2}$ and a good choice is resulted a quartz cell, with infrasil windows, containing a mixture of the halogen-hydrates $\mathrm{HCl}, \mathrm{HBr}$ and $\mathrm{HI}$.

The presence of $\mathrm{HI}$ is cause of some practical problems connected to the fact that $\mathrm{HI}$ dissociates for either high temperatures (above $500{ }^{\circ} \mathrm{C}$ ), or when exposed to light of wavelength lower than $500 \mathrm{~nm}$. As the cell is made by quartz, and must be sealed by using a high temperature flame, it is evident that some HI will be lost in this procedure. The problem is then to minimize the effects of sealing, and to check the effective amount of HI inside the cell.

We give here a description of the mechanics and of the optics of the cell, with particular attention to the filling and sealing procedure.

We present also a characterization of the absorption spectra of the mixture, contained in the cell, by means of an Infrared Fourier Transform Spectrometer Bruker present at LENS (European Laboratory for Non-linear Spectroscopy) in Florence (Italy).

* oliva@arcetri.astro.it; ; phone ++39055 27521; fax ++ 39055220039 ; www.arcetri.astro.it 


\section{DESIGN OF THE ABSORPTION CELL}

Details of the quartz cell will be given in the following. Here we focus on the mechanics, whose aim is twofold: to provide the insulation vacuum, and to insure the thermal dissipation for the Peltier coolers. The container for the insulation vacuum is made by a Stainless Steel (SS) pipe (101.6 $\mathrm{mm} \mathrm{OD,} 3.05 \mathrm{~mm}$ thickness), holding vacuum flanges at both ends. The pipe is closed by two disks, with $\mathrm{ZnSe}$ windows in their centers, to allow the light propagation, while stopping wavelengths shorter than yellow. The electrical feedthrough and the evacuation pipe are located in one of the disk. The dimensions of the vacuum vessel, excluding vacuum valve and feedthrough, are: length $532 \mathrm{~mm}$, diameter 124 $\mathrm{mm}$. A photo of the cell (with aluminum disks in place of $\mathrm{ZnSe}$ windows) is in Fig. 1 .

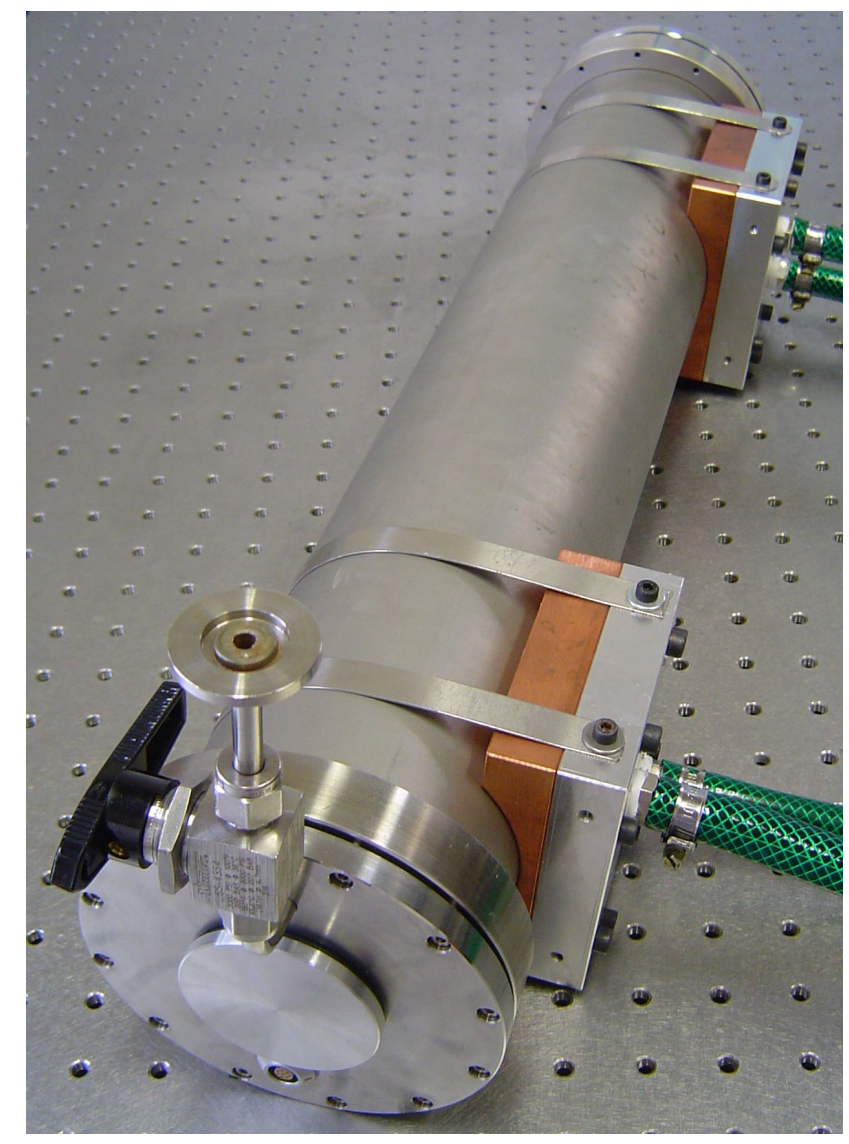

Fig. 1. Picture of the cell. The ZnSe windows are temporarily replaced with aluminum disks, to stop light completely.

The problem of temperature is to be divided in two parts. First of all, it is necessary to efficiently remove heat from the quartz pipe, towards the SS pipe. It is important to know that, under vacuum, the use of heat conductive greases is to be avoided. So the thermal contact between the Peltier elements (Melcor CP1.4-127-045L) and the copper blocks must occur first of all because of the surfaces flatness. In order to reach this result, the copper blocks have been flattened and polished, close to optical quality. Moreover, the nylon screws have been located in suitable positions to exert the best pressure, according to the datasheet of the Peltier elements. In order to keep the reservoir copper block at constant temperature, even below room temperature, so making it easier to cool down the inner cell, two heat removers are located outside the SS pipe, in which a cooling fluid (water or ethylene glyicol) can be flushed.

Finally, the thermal insulation of the quartz cell is increased by rolling around it a film of aluminated mylar.

All these precautions, plus a PID regulator (CAL Controls Ltd, mod. 3200), allow a temperature stability of $0.4{ }^{\circ} \mathrm{C}$, down to $-20^{\circ} \mathrm{C}$. 


\section{FILLING AND SEALING PROCEDURE OF THE CELL}

As already discussed in a previous paper ${ }^{2}$, the choice of gas mixture must fulfill several conditions. First of all the gases inside the cell must have strong and uniformly distributed absorption bands all over the instrumental range. The bands must be well resolved, without interference or overlap with other atmospheric absorptions. The intrinsic width of the absorption lines should be lower than the resolution of the spectrometer. The molecules must also have the largest possible dipole momentum so that the first, and stronger, overtones fall in the wavelength range of interest. Moreover, the temperature of absorbing gas should be lower than $-20^{\circ} \mathrm{C}$, in order to avoid ambient black-body emission in coincidence with the absorption lines, and consequently the gas mixture must consist of chemical species with a low boiling temperature (lower than $-20^{\circ} \mathrm{C}$ ). Finally the molecules should be easy to handle and stable with time.

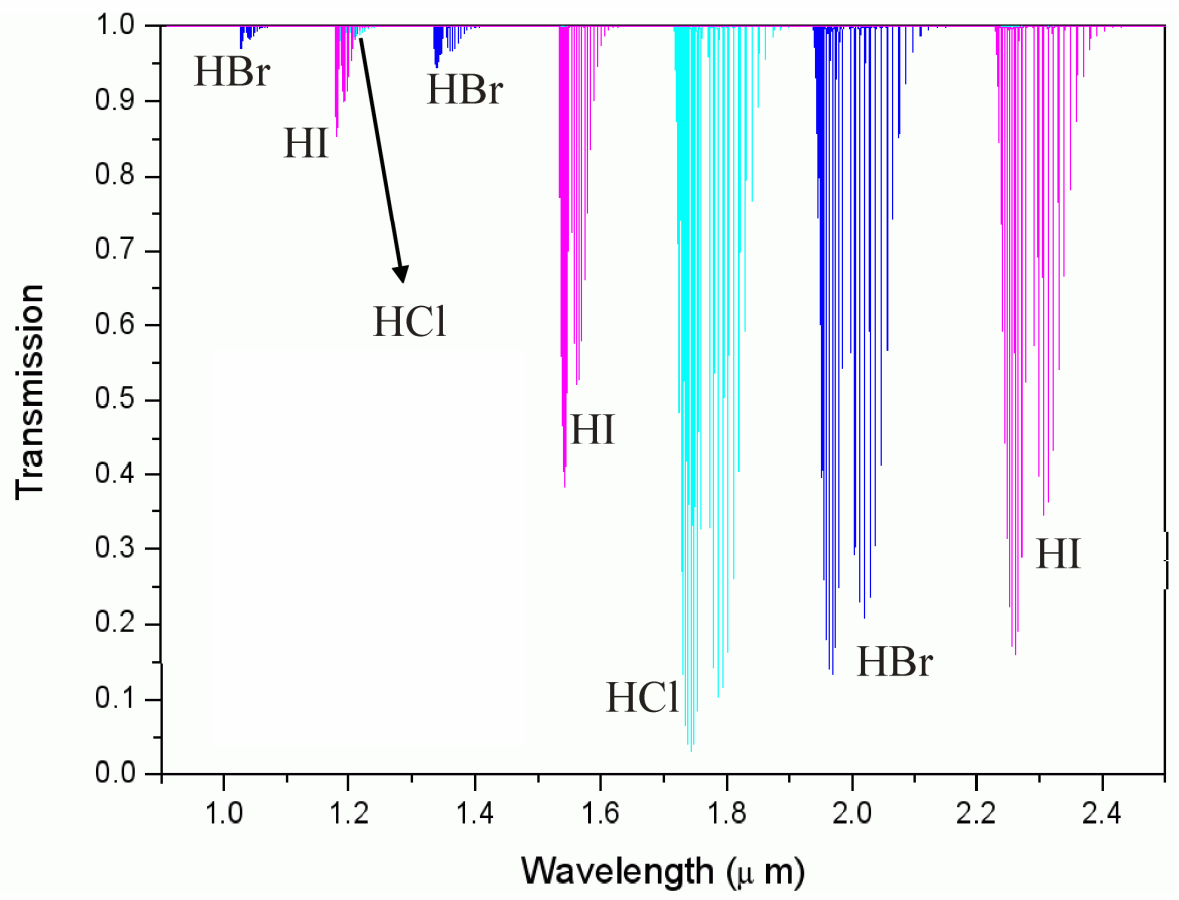

Fig. 2. Theoretical absorption spectrum of $\mathrm{HCl} 5 \%, \mathrm{HBr} 55 \%$ and $\mathrm{HI} 40 \%$, for a path-length of $0.5 \mathrm{~m}$, a pressure of 1 atm and a temperature of $253 \mathrm{~K}$. The spectrum is computed using the HITRAN database.

A good choice is that to use a mixture of three hydrides of halogen gases with the following concentrations: $\mathrm{HCl} 5 \%$, $\mathrm{HBr} 55 \%$ and $\mathrm{HI} 40 \%$. In such a way we obtain a mixture with more than two hundreds absorption lines in the range $0.9-2.5 \mu \mathrm{m}$.

In Fig. 2 we show the theoretical absorption spectrum of the mixture computed using the HITRAN ${ }^{3}$ database and rebinned to the GIANO resolving power of $\mathrm{R}=5 \cdot 10^{4}$. Starting from the longer wavelengths, the first three groups of prominent lines correspond to the $\Delta \mathrm{v}=2$ roto-vibrational transitions of $\mathrm{HI}(\sim 2.3 \mu \mathrm{m}), \mathrm{HBr}(\sim 2.0 \mu \mathrm{m})$ and $\mathrm{HCl}(\sim 1.7 \mu \mathrm{m})$. The following are the second overtone of $\mathrm{HI}(\sim 1.5 \mu \mathrm{m})$ and $\mathrm{HBr}(1.3 \mu \mathrm{m})$; the third overtone of $\mathrm{HI}(\sim 1.2 \mu \mathrm{m}$, overlapped with the weaker second overtone of $\mathrm{HCl})$ and $\mathrm{HBr}(\sim 1.05 \mu \mathrm{m})$.

It should be noted that Chlorine has two isotopes with natural abundances in the ratio $3: 1$ (weights 35 and 37, respectively), while Bromine has the two isotopes 79 and 81 in a 1:1 ratio. So for $\mathrm{HCl}$ and $\mathrm{HBr}$ each band has two series of lines. 
All the gases $\mathrm{HCl}, \mathrm{HBr}$ and $\mathrm{HI}$ are corrosive, but they cannot attack quartz and do not present particular handling problems in absence of water.

Problems arise from the fact that $\mathrm{HI}$ dissociates for either temperatures higher than $500{ }^{\circ} \mathrm{C}$, or when exposed to UV radiation. As the alternative to HI would have been HF, we have preferred to face the problems of finding a supplier of $\mathrm{HI}$, and keeping it in safe conditions, rather than changing gas, to a much more problematic one.

Before closure, the cell appears like in Fig. 3. The valve is made by pyrex and has a Teflon sealing, suitable for operation with such a mixture. It has been glued to the quartz by using Torr-Seal ${ }^{\mathrm{TM}}$. A metallic pipe is glued to the valve, again by using Torr-Seal ${ }^{\mathrm{TM}}$, and this pipe is connected to the vacuum line for the filling of the cell. The vacum line consists of three lines connected to the gas cylinders, a line connected to the pipe of the cell, a line connected to a liquid-nitrogen trap in conjunction with a rotary pump, and a manometer to measure the pressure. Each line is separated from the others with a valve.

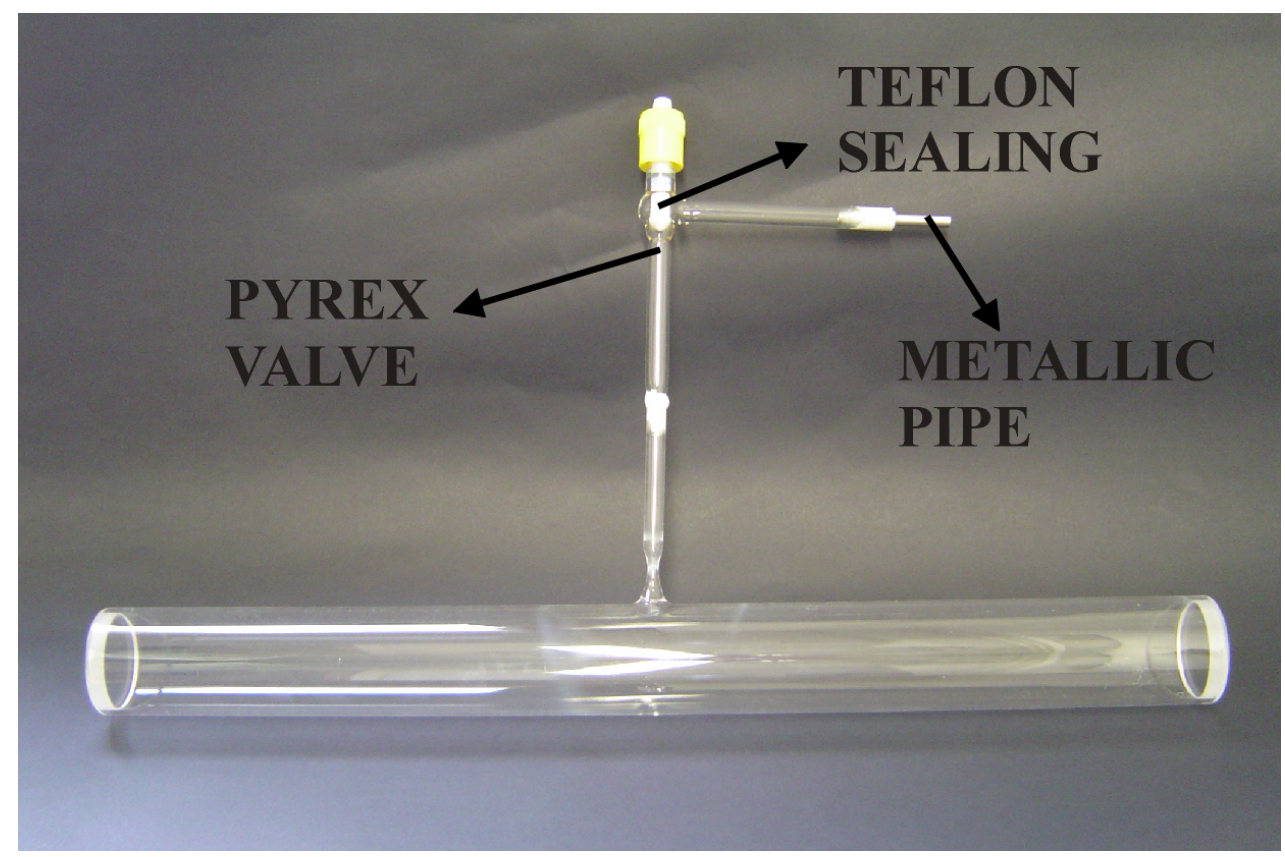

Fig. 3. Absorption cell before closure

The diameter of the quartz pipe of the cell, connected to the pyrex valve, is reduced very close to the cell, to allow the sealing of the cell with a high temperature flame, after the filling procedure.

$\mathrm{HCl}$ and $\mathrm{HBr}$ are stable gases and easy to find on the market with the required purity. As said before, $\mathrm{HI}$ can be easily dissociated by UV radiation. For this reason it is very difficult (and expensive) to find in the form of pure gas, and must be carefully shielded against UV radiation once inserted into the cell.

The filling procedure has been carried out and the pressure of the gases inside the cell are: $45 \mathrm{mBar}$ for $\mathrm{HCl}, 505 \mathrm{mBar}$ for $\mathrm{HBr}$, and $400 \mathrm{mBar}$ for $\mathrm{HI}$. The total pressure is slightly below $1 \mathrm{~atm}$ (about $950 \mathrm{mBar}$ ), in order to allow the implosion of the melted quartz.

During the procedure of cell sealing by using a high temperature flame, it is unavoidable that some HI will be lost. In order to reduce the problems of sealing, we have covered the pipe surface with black hard paper, and then with an aluminum foil. In this way, the only part of the cell exposed to light is the narrow pipe to be cut. As for heating, the time necessary for sealing is less than 20 seconds, so the cell has no time to heat above $500{ }^{\circ} \mathrm{C}$.

During the first attempt to close the cell, we have obtained an unwanted, and very dangerous effect for the mixture: the $\mathrm{HI}$ in the region between the sealing point and the valve has been dissociated, and iodine has started a bright emission, 
just like in projectors for cars. The white light emitted could enter the cell without any filter and this effect, together with heating in proximity of the sealing point, has led to the dissociation of about $70 \%$ of the HI.

We studied how to overcome this effect, and we decided to exploit the relatively high melting or boiling temperatures of these gases. Table 1 lists the boiling and melting temperatures of the three acids, plus those of nitrogen and $\mathrm{CO}_{2}$. Unless differently specified, data are taken from the "Gas Encyclopædia" by Air Liquide, Ed. Elsevier.

Table 1. Melting and boiling temperatures for some gases

\begin{tabular}{|l|l|l|}
\hline Gas & Melting $\mathrm{T}\left({ }^{\circ} \mathrm{C}\right)$ & Boiling $\mathrm{T}\left({ }^{\circ} \mathrm{C}\right)$ \\
\hline $\mathrm{HCl}$ & -114.8 & -85.1 \\
\hline $\mathrm{HBr}$ & $-88.6\left(\right.$ WebElements ${ }^{\mathrm{TM}}$ Periodic table) & -66.8 \\
\hline $\mathrm{HI}$ & -51 & -35.4 \\
\hline $\mathrm{N}_{2}$ & -210 & -195.9 \\
\hline $\mathrm{CO}_{2}$ & -78.5 (Sublimation) & \\
\hline
\end{tabular}

The idea is to freeze as much as possible of the acids, in particular HI, far from the sealing point. In this way no dissociation due to temperature could take place, and photo-dissociation should be due only to that light, guided by the pipe walls up to the cold finger. Moreover, the "iodine projector" effect would be completely avoided.

In order to create a cold finger at a sufficiently low temperature, we should use liquid nitrogen. Yet, even if the thermal expansion coefficient of quartz is very small $(\approx 0.5 \mathrm{ppm} / \mathrm{K})$, the boiling temperature of nitrogen could be such to induce breaks in case of stressed material. The sublimation temperature of $\mathrm{CO}_{2}$ is low enough to freeze $\mathrm{HI}$ (which is the main goal of this procedure), and to liquefy $\mathrm{HBr}$. The density of $\mathrm{HCl}$ in the seal region would be substantially reduced. So we have decided to use dry ice.

The sealing procedure has occurred according to our forecasts. No "iodine projector" effect was observed, which means that HI was frozen indeed, far from the sealing point. We have checked the content of the cell before and after closure, and no difference was observed within the experimental errors.

\section{CHARACTERIZATION OF THE CELL BY MEANS OF A FOURIER TRANSFORM SPECTROMETER}

After the sealing procedure, a characterization of the absorption spectra of the mixture, contained in the cell, was carried out by means of an Infrared Fourier Transform (FTIR) Spectrometer (Bruker IFS 120-HR), present at LENS in Florence (Italy), covering the $10-25000 \mathrm{~cm}^{-1}$ spectral range with a maximum resolution (in the Far Infrared region) of $0.002 \mathrm{~cm}^{-1}$.

In Fig. 4 we show the spectrum obtained with a resolution of $0.1 \mathrm{~cm}^{-1}$, by using a GLOBAR lamp as source, a quartz beam-splitter and a InSb detector. The cell was inserted in the spectrometer in a vertical position, so that the radiation path-length is not $50 \mathrm{~cm}$ but only $4.6 \mathrm{~cm}$, that is the diameter of the cell. So the absorption signals are reduced of a factor of about 11. In spite of this reduction, the presence in the mixture of $\mathrm{HCl}, \mathrm{HBr}$ and $\mathrm{HI}$ is clearly demonstrated.

The oscillating background of Fig. 4 is due to the instrument and could be removed with a fitting procedure. 


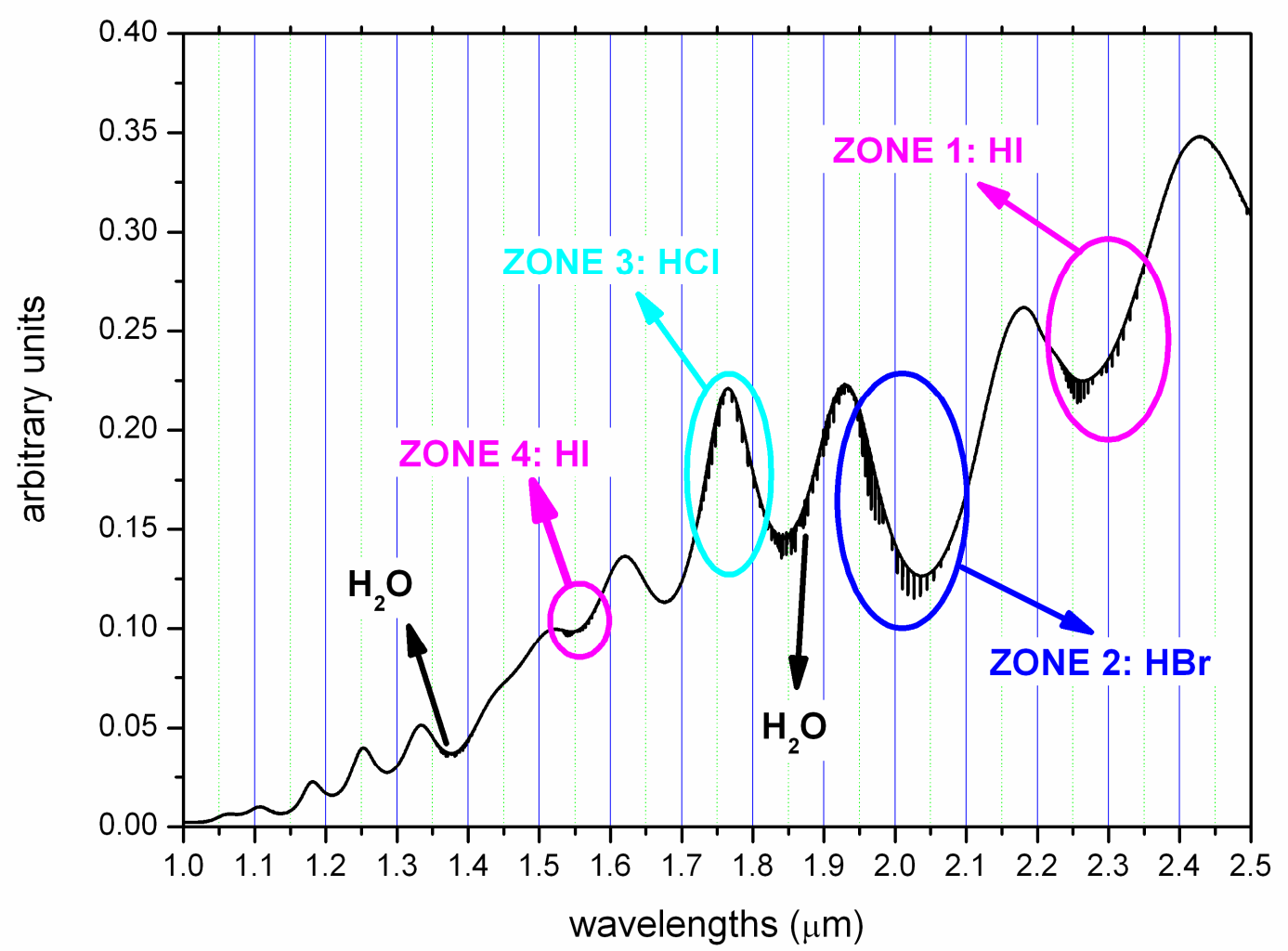

Fig. 4. Absorption spectra of the mixture contained in the cell $(\mathrm{HCl}, \mathrm{HBr}$ and $\mathrm{HI})$, after the sealing procedure, obtained with an Infrared Fourier Transform Spectrometer (Bruker IFS 120-HR), with a resolution of $0.1 \mathrm{~cm}^{-1}$. Four regions, corresponding to mixture absorptions, are clearly identified inside the circles. The other absorption signals, outside the circles, are due to the presence of water absorption lines. The oscillating background is due to the instrument

The reduction of the absorption signals, due to the smaller path-length radiation, allow to observe only the $\Delta \mathrm{v}=2$ rotovibrational transitions of $\mathrm{HI}$ (zone 1 in Fig. 4), $\mathrm{HBr}$ (zone 2 in Fig. 4) and $\mathrm{HCl}$ (zone 3 in Fig. 4) and the second overtone of HI (zone 4 in Fig. 4). The other absorption signals shown in Fig. 4 are in conjunction with water absorption lines.

A zoom of the single zones inside the circles of Fig. 4 is shown in Fig. 5. 

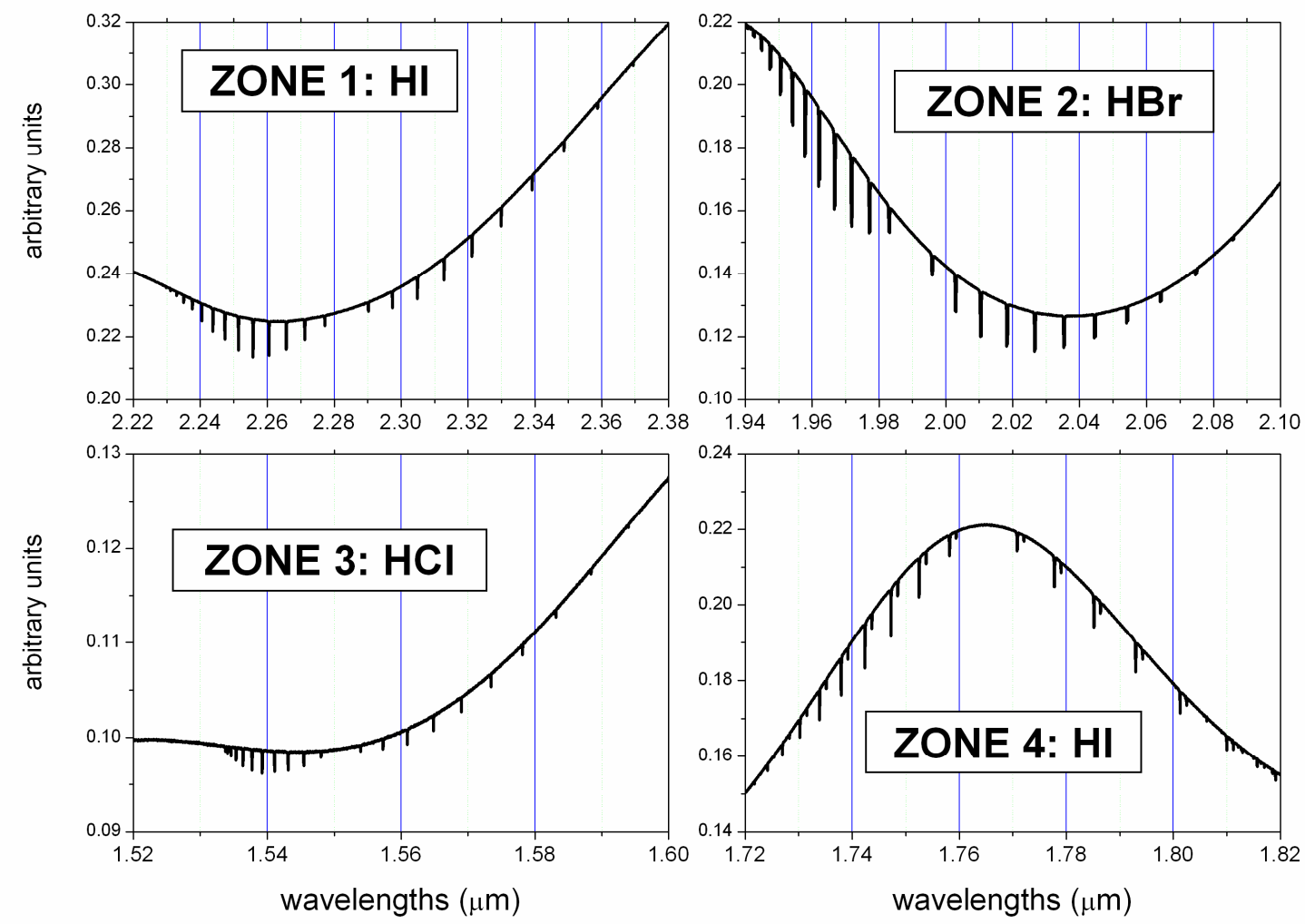

Fig. 5. Absorption spectra of the mixture contained in the cell $(\mathrm{HCl}, \mathrm{HBr}$ and $\mathrm{HI})$ obtained with an Infrared Fourier Transform Spectrometer (Bruker IFS 120-HR), with a resolution of $0.1 \mathrm{~cm}^{-1}$. Each plot is a different zoom of the signal shown in Fig. 4. The oscillating background is due to the instrument.

\section{CONCLUSIONS}

We have described the laboratory work for the construction of an absorption cell, containing a mixture of $\mathrm{HCl}, \mathrm{HBr}$ and $\mathrm{HI}$, useful as a reference for radial velocity measurements with accuracy of few $\mathrm{m} / \mathrm{s}$. This cell will be part of the GIANO spectrometer.

A description of the mechanics and of the optics of the cell has been presented.

We have also shown the precautions we have used during filling and sealing procedure with high temperature flame, in order to solve the practical problems connected to the fact that $\mathrm{HI}$ dissociates for either temperatures above $500{ }^{\circ} \mathrm{C}$ or when exposed to light of wavelength lower than $500 \mathrm{~nm}$. The idea to use dry ice to freeze as much as possible of the acids, in particular HI, far from the sealing point has been successful.

After the sealing procedure, the presence inside the cell of $\mathrm{HI}, \mathrm{HCl}$ and $\mathrm{HBr}$ has been demonstrated by recording the absorption spectra of the mixture with an Infrared Fourier Transform Spectrometer.

\section{AKNOWLEDGEMENT}


The authors wish to thank Mr. Paolo Bianchi and Mr. Massimo D'Uva of the CNR-INOA (Istituto Nazionale di Ottica Applicata, Florence, Italy) for their contribution in the realization of the optics and of mechanics of the cell.

The authors are also very grateful to Mr. Mino Badalassi of the CNR - IPCF (Istituto per i Processi Chimico-Fisici, Pisa, Italy) for his very effective support in the realization and in the sealing procedure of the quartz cell.

The cooperation of Prof. R. Bini and Dr. Margherita Citroni for the FTIR spectra at LENS (European Laboratory for Non-Linear Spectroscopy, Florence, Italy) is also gratefully acknowledged.

\section{REFERENCES}

[1] Oliva, E., Origlia, L., Baffa, C., Biliotti, V., Bruno, P., D’Amato, F., Del Vecchio, C., Falcini, G., Gennari, S., Ghinassi, F., Giani, E., Gonzalez, M., Leone, F., Lolli, M., Lodi, M., Maiolino, R., Mannucci, F., Marcucci, G., Mochi, I., Montegriffo, P., Rossetti, E., Scuderi, S. and Sozzi, M., “The GIANO-TNG spectrometer", Proc. SPIE 6269, 626919 (2006).

[2] D'Amato, F., Oliva, E. and Origlia, L., "The gas absorption cell for GIANO-TNG spectrometer", Proc. SPIE 6269, 62695E (2006).

[3] Rothman, L. S., Jacquemart, D., Barbe, A., Chris Benner, D., Birk, M., Brown, L. R., Carleer, M. R., Chackerian Jr., C., Chance, K., Coudert, L. H., Dana, V., Devi, V. M., Flaud, J.-M., Gamache, R. R., Goldman, A., Hartmann, J.M., Jucks, K. W., Maki, A. G., Mandin, J.-Y., Massie, S. T., Orphal, J., Perrin, A., Rinsland, C. P., Smith, M. A. H., Tennyson, J., Tolchenov, R. N., Toth, R. A., Vander Auwera, J., Varanasi, P. and Wagner, G., "The HITRAN 2004 molecular spectroscopic database", J. Quant. Spectrosc. Radiat. Transf. 96, 139 (2005). 